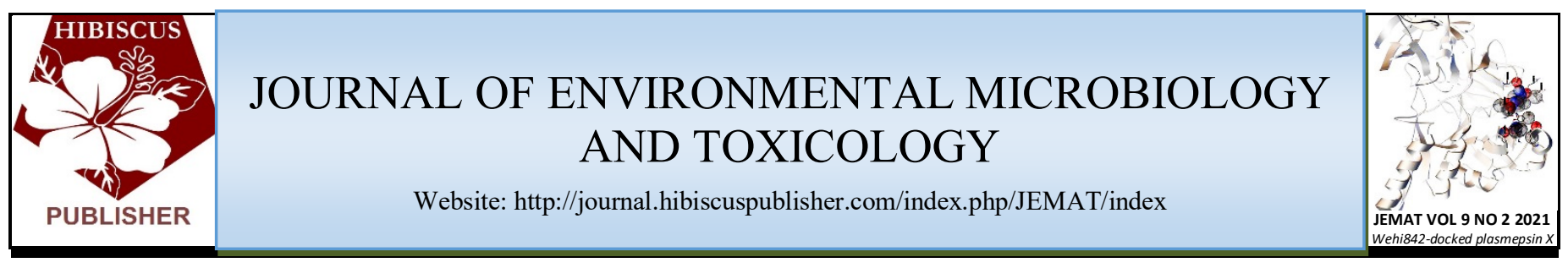

\title{
Detection Limit of the Four-Parameter Logistic Model for the Quantitative Detection of Serum Squamous Cell Carcinoma Antigenin Cervical Cancer Based on Surface Plasmon Resonance Biosensor
}

\author{
Noor Azlina Masdor ${ }^{1 *}$ \\ ${ }^{1}$ Biotechnology Research Centre, Malaysian Agricultural Research and Developmental Institute (MARDI), \\ 43400 Serdang, Selangor, Malaysia. \\ *Corresponding author: \\ Dr. Azlina Masdor, \\ Biotechnology Research Centre, \\ Malaysian Agricultural Research and Developmental Institute (MARDI), \\ 43400 Serdang, \\ Selangor, \\ Malaysia. \\ Email: azlina@mardi.gov.my
}

\section{HISTORY \\ Received: $15^{\text {th }}$ Oct 2021 \\ Received in revised form: $28^{\text {th }}$ Nov 2021 Accepted: $13^{\text {th }}$ Dec 2021}

\section{KEYWORDS \\ Limits of Detection \\ Four-parameter logistic \\ Biosensor \\ Cervical cancer \\ Surface Plasmon Resonance}

\begin{abstract}
Biochemical diagnostic procedures, such as protein binding, rely on biomolecular interactions as its diagnostic modality, and as a consequence, their calibration curves are more complex. In addition, sigmoidal curves are often seen in these tests. In the event of asymmetry, a logistic (5PL) curve, or a logistic (4PL) curve, may be the best way to represent a distinctive sigmoidal relationship. It is possible that the linearization of an otherwise nonlinear connection by $\log$ transformation may result in a disruption of the error structure of the curve, and that this will have the opposite effect of decreasing or even eliminating error in the relationship. Previously, a surface plasmon resonance biosensor for the detection of squamous cell carcinoma antigen (SCCa) using nanoparticle technology was developed. However, based on the calibration curve, it conforms to the majority on sigmoidal shape curve for antibody-type sensing system. The resultant curve showed a sigmoidal calibration curve but was not modelled according to any of the sigmoidal models available. The LOD value obtained through the 4PL modelling exercise based on the classical method was $0.255 \mathrm{pM}$ (95\% confidence interval of 0.167 to 0.379 ) while the pooled standard deviation (PSD) method yielded an LOD value of $0.035 \mathrm{pM}(95 \%$ confidence interval of 0.011 to 0.067 ), which indicates that the PSD method was superior.
\end{abstract}

\section{INTRODUCTION}

A major drawback of the current literature on bioassay development is that these tests are not made using statistically robust methods for establishing the limit of detection. As an alternative, researchers often make use of simple detection-limit methods that are only roughly indicative of the actual detection limit. We can only assume that this is due to a practical need for simplified processes, in addition to the notion that the limit of detection theory has already been lowered to practise for bioassays. The present literature on bioassay development does not make use of statistically robust techniques for determining the limit of detection of a particular test, which is a significant limitation. Instead, researchers often use basic techniques that give an approximate estimate of the detection limit, sometimes without any indication of confidence in the estimate. This lack of robust techniques is likely owing to a practical desire for easy and accessible procedures, as well as a lack of such approaches that have reduced the ideas of limit of detection theory to practise for bioassays.
Cervical cancer is an international public health problem that affects women of all ages. The illness is the second most common cancer in women globally, and it is the fourth greatest cause of cancer-related deaths among women $[1,2]$. FIGO reports that cervical cancer has a 5-year recurrence rate of 28 percent and a 5 -year total death rate of 27.8 percent, respectively, among women who have had the disease for five years. According to Muoz et al., squamous cell carcinoma (SCC) is the most frequent histological form of cervical cancer, accounting for $60-80$ percent of all cases [3].

It was Kato and Torigoe that discovered Squamous cell carcinoma antigen (SCCa), a glycoprotein with isoforms varying in size from 45 to $55 \mathrm{kDa}$, and identified it as a tumor-associated antigen for the first time[4,5]. Since its discovery, SCCa has been discovered to be raised in a variety of SCCs, including those of the uterine cervix, lung, oral cavity, skin, oesophagus, head and neck, oesophagus, anal canal, and vulva [6,7]. Patients with SCC of the uterine cervix are increasingly using SCCa as a particular tumour marker for immunostimulatory diagnosis, predictive risk appraisal, treatment monitoring, and follow-up of recurrence in 
order to improve their overall survival [8]. Nevertheless, the clinical application of SCCa as a diagnostic marker for cervical cancer initial detection until care is seriously constrained by the huge disparity in detectability, which varies from 28 percent to 88 percent as per various studies, based on a variety of inclusion criteria, inconsistent cut-off values, and methodological defects [9]. Previously, a surface plasmon resonance biosensor for the detection of squamous cell carcinoma antigen ( $\mathrm{SCCa}$ ) using nanoparticle technology was developed. However, based on the calibration curve, it conforms to the majority on sigmoidal shape curve for antibody-type sensing system. The resultant curve showed a sigmoidal calibration curve but was not modelled according to any of the sigmoidal models available [10]. The objective of this study is the remodel the data using the standard 4-PL model and to determine the Limits of Detection (LOD) based on the two methods; the classical definition of limits of detection method and the method based on pooled standard deviation (PSD).

\section{Acquisition of Data}

Data from a published work [10] from figure 3 showing a calibration curve of GMO detection on the microfluidic disk from the GMO in a certified reference materials; maize Bt11 (ERMBF412b and ERM-BF412f). The data were processed using the software Webplotdigitizer 2.5 [11] which digitizes the scanned figure into a comma separated value [12].

\section{Four parameter logistics modelling}

In the assay, the calibration curves will be fitted with a non-linear regression using a four-parameter dose-response equation $[13,14]$ as following:

$$
y=\frac{a-d}{1+\left(\frac{x}{c}\right)^{b}}+d
$$

Where $y$ signifies the response signal (optical density), $\boldsymbol{x}$ signifies the DNA log concentration, $\boldsymbol{a}$ and $\boldsymbol{d}$ signify the maximum and minimum signal response of the calibration curve, correspondingly, $\boldsymbol{b}$ is the Hill coefficient which represents the slope-like parameter and $c$ represents the DNA log concentration producing a $50 \%$ signal response $\left(\mathrm{EC}_{50}\right)$ value. Both the classical three times the standard deviation of the blank and another statistically robust technique for estimating the analytical LOD of a classic sigmoidal correlation based on the pooled standard deviation of datapoints will be utilized [15]. In the traditional LOD determination, a single test concentration which is usually taken from the lowest tested concentration or control will be utilized to obtain the standard deviation of the blank.

Most assays are carried out using fewer than 10 replicates per concentration with the norm is three replicates per concentration level. Anything less than 10 replicates per concentration have been shown to give a less accurate representation of the population variance and recommendation is to pool the standard deviation for all test samples [15]. To account for the variability in variance when there are less than 10 replicates per concentration level, the data points can be weighted by inverse variance. Due to the fact that this strategy is not suggested when there are fewer than 10 replicates per concentration level, an unweighted fit was utilised in this particular instance instead. In addition, it should be emphasised that the unweighted fit is consistent with the assumption of homoscedasticity that was established for the pooled estimate of variance that was originally applied. The limit of detection (LOD) will be determined by calculating the mean value of absorbance at a blank concentration of DNA log concentration at three successive PSDs. For the purpose of calculating the LOD and performing regression analysis, the four-parameter logistics model and non-linear regression analysis software will be used in conjunction (PRISM, v 5.1) from www.graphpad.com.

\section{RESULT AND DISCUSSION}

ELISA-based and biological receptors-based or DNA-based standard curves are generally nonlinear and sigmoidal in property, and the best way to fit this kind of curve is to use a standard four-parameter logistic (4-PL) or the rarely used five parameter logistic (5-PL) models [16]. The raw data should then be fitted to the 4-PL curve through a modification of the curve model's parameters to achieve an ideal fitting between experimental and calculated data; the latter is often represented by a line running through the experimental data [17]. Although a patently sigmoidal profile was obtained by [10], the authors reported that the detection limits (LODs), calculated as the lowest amount of DNA that is able to produce a signal distinguished from the blanks were between 110 to $460 \mathrm{mg} / \mathrm{g}(0.011-0.046 \%)$. The result in Fig. 1. shows a typical sigmoidal curve for the calibration curve based on the 4-PL equation. A typical sigmoidal profile was obtained. A good correlation coefficient value of 0.965 was obtained indicating good fitting.

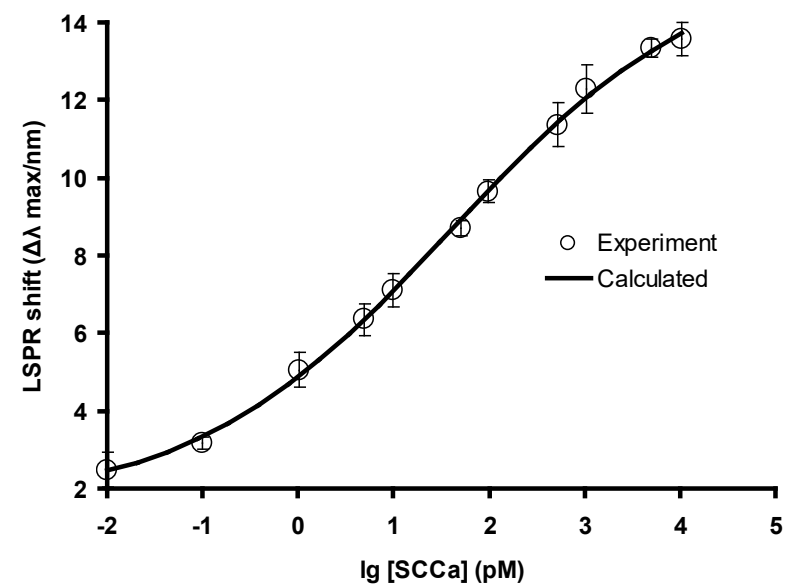

Isothermal solid-phase recombinase polymerase amplification on microfluidic digital versatile discs.

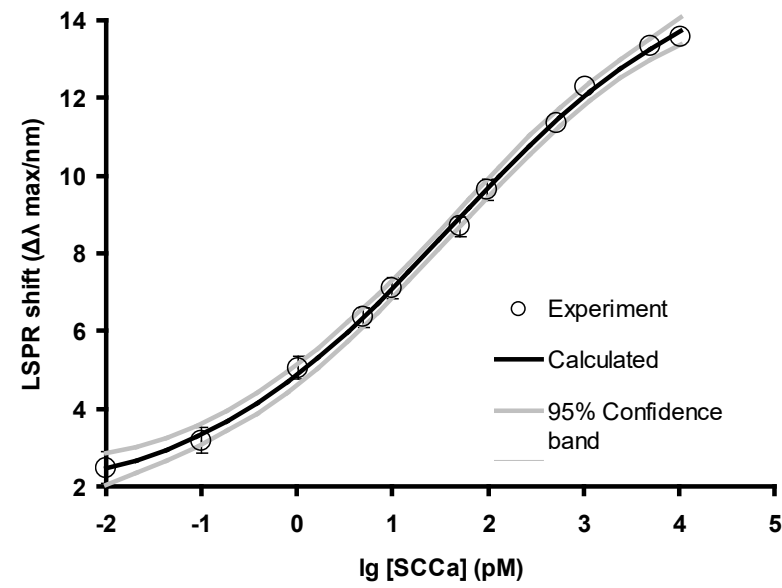

Isothermal solid-phase recombinase polymerase amplification on microfluidic digital versatile discs calibration curve, and its $95 \%$ confidence band. 
The value of the curve parameters is shown in the form of the four-parameter logistic equation as follows;

$y=1.512+\frac{14.25}{1+10^{(1.602-x) * 0.3201}}$

The LOD value obtained through the 4PL modelling exercise based on the classical method was $0.255 \mathrm{pM}(95 \%$ confidence interval of 0.167 to 0.379 ) while the PSD method yielded an LOD value of $0.035 \mathrm{pM}$ (95\% confidence interval of 0.011 to 0.067 ), which indicates that the PSD method was superior. The classical method was higher than the rough estimation employed by [10] with an LOD value of $0.125 \mathrm{pM}$. Because it is recommended that LOD values be computed using the 4-PL technique in the case that the curve has an obviously sigmoidal profile, the LOD value derived using the 4PL modelling approach should be utilised to report the LOD value in this situation.

Last but not least, in the event that the calibration curve has a sigmoidal profile, the 4PL model should be used to fit the data rather than a linear model, and the LOD value should be derived using the 4PL model rather than a linear model. According to the findings of this study, the adoption of the 4Pl model proved successful, as it was able to represent the entire date curve rather than just a linear section of it. When developing an ELISA technique, the linear part is crucial since it is a handy and speedy approach for determining the sensitivity of the method. It is also typically a more beneficial method in field applications when a quick and easy assessment is required. The 4Pl model, on the other hand, should not be abandoned because it is capable of reporting the LOD value and its 95 percent confidence range for any created technique with high accuracy.

\section{ACKNOWLEDGEMENT}

This work was supported by MARDI via WRM fund (TP-RB0023).

\section{REFERENCES}

1. Woodman CB, Collins SI, Young LS. The natural history of cervical HPV infection: unresolved issues. Nat Rev Cancer. 2007;7(1):11-22.

2. Li J, Li LK, Ma JF. Knowledge and attitudes about human papillomavirus (HPV) and HPV vaccines among women living in metropolitan and rural regions of China. Vaccine. 2009;27(8):1210-5.

3. Muñoz N, Bosch FX, Sanjosé S. International Agency for Research on Cancer Multicenter Cervical Cancer Study Group. Epidemiologic classification of human papillomavirus types associated with cervical cancer. N Engl J Med. 2003;348(6):51827.

4. Kato H, Torigoe T. Radioimmunoassay for tumor antigen of human cervical squamous cell carcinoma. Cancer. 1977;40(4):1621-8.

5. Kato H, Morioka H, Aramaki S, Torigoe T. Radioimmunoassay for tumor-antigen of human cervical squamous cell carcinoma. Cell Mol Biol Incl Cyto Enzymol. 1979;25(1):51-6.

6. Ho YJ, Hsieh JF, Tasi SC, Lee JK, Kao CH. Tissue polypeptide specific antigen and squamous cell carcinoma antigen for early prediction of recurrence in lung squamous cell carcinoma. Lung. 2000;178(2):75-80.

7. Bandoh N, Ogino T, Katayama A. HLA class I antigen and transporter associated with antigen processing downregulation in metastatic lesions of head and neck squamous cell carcinoma as a marker of poor prognosis. Oncol Rep. 2010;23(4):933-9.

8. Zhao Q, Feng Y, Mao X, Qie M. Prognostic value of fluorine-18fluorodeoxyglucose positron emission tomography or PETcomputed tomography in cervical cancer: a meta-analysis. Int $\mathrm{J}$ Gynecol Cancer. 2013;23(7):1184-90.
9. Gadducci A, Tana R, Cosio S, Genazzani AR. The serum assay of tumour markers in the prognostic evaluation, treatment monitoring and follow-up of patients with cervical cancer: a review of the literature. Crit Rev Oncol Hematol. 2008;66(1):10-20.

10. Zhao Q, Duan R, Yuan J, Quan Y, Yang H, Xi M. A reusable localized surface plasmon resonance biosensor for quantitative detection of serum squamous cell carcinoma antigen in cervical cancer patients based on silver nanoparticles array. IJN. $2014 \mathrm{Feb}$ 22;9(1):1097-104.

11. Rohatgi A.

WebPlotDigitizer. http://arohatgi.info/WebPlotDigitizer/app/ Accessed June 2 2014.; 2015.

12. Khare KS, Phelan Jr FR. Quantitative comparison of atomistic simulations with experiment for a cross-linked epoxy: A specific volume-cooling rate analysis. Macromolecules. 2018;51(2):56475.

13. Iturria SJ. Statistical inference for relative potency in bivariate dose-response assays with correlated responses. J Biopharm Stat. 2005; 15(2):343-51

14. Masdor NA, Altintas Z, Shukor MY, Tothill IE. Subtractive inhibition assay for the detection of Campylobacter jejuni in chicken samples using surface plasmon resonance. Scientific Reports. 2019 Sep 20;9(1):13642.

15. Holstein CA, Griffin M, Hong J, Sampson PD. Statistical method for determining and comparing limits of detection of bioassays. Anal Chem. 2015 Oct 6;87(19):9795-801.

16. Masdor NA, Altintas Z, Tothill IE. Sensitive detection of Campylobacter jejuni using nanoparticles enhanced QCM sensor. Biosensors and Bioelectronics. 2016;78:328-36.

17. Cumberland WN, Fong Y, Yu X, Defawe O, Frahm N, De R. Nonlinear Calibration Model Choice between the Four and FiveParameter Logistic Models. Journal of Biopharmaceutical Statistics. 2015;25(5):972-83. 